\title{
1 Selection for Gaia across multiple scales
}

2 Timothy M. Lenton ${ }^{1}$, Stuart J. Daines ${ }^{1}$, James G. Dyke ${ }^{2}$, Arwen E. Nicholson ${ }^{1}$, David M.

3 Wilkinson ${ }^{3}$, Hywel T. P. Williams $s^{1,4}$

$4 \quad{ }^{1}$ Earth System Science, College of Life and Environmental Sciences, University of Exeter, EX4

$5 \quad 4 Q E, U K$

$6 \quad{ }^{2}$ Geography and Environment, University of Southampton, SO17 1BJ, UK

$7 \quad{ }^{3}$ School of Life Sciences, University of Lincoln, LN6 7DL, UK

$8{ }^{4}$ Computer Science, College of Engineering, Mathematics and Physical Sciences, University

9 of Exeter, EX4 4QE, UK

10 *Corresponding author: Lenton, T. M. (t.m.lenton@exeter.ac.uk)

11 Keywords

12 Gaia hypothesis; environmental regulation; feedback; selection; biogeochemical cycling;

13 climate 
15

\section{Abstract}

Recently postulated mechanisms and models can help explain the enduring "Gaia" puzzle of environmental regulation mediated by life. Natural selection can produce nutrient recycling at local scales and regulation of heterogeneous environmental variables at ecosystem scales. However, global-scale environmental regulation involves a temporal and spatial decoupling of effects from actors that makes conventional evolutionary explanations problematic. Instead, global regulation can emerge by a process of "sequential selection" in which systems that destabilize their environment are short-lived and result in extinctions and reorganizations until a stable attractor is found. Such persistence-enhancing properties can in turn increase the likelihood of acquiring further persistence-enhancing properties through "selection by survival alone". Thus, Earth system feedbacks provide a filter for persistent combinations of macro-evolutionary innovations.

\section{The Gaia puzzle}

The Gaia hypothesis [1-3] posits that a coupled system of life on Earth and its abiotic environment self-regulates in a habitable state, despite destabilizing influences such as a steadily brightening Sun, changing volcanic, metamorphic and tectonic activity, and occasional massive meteorite impacts [3, 4]. Environmental regulation is postulated to include the stabilisation of atmospheric composition and climate $[1,3,4]$, ocean nutrients [5-8], and the global (re)cycling of essential elements for life $[9,10]$. Furthermore, the environmental state that is regulated is suggested to have changed over Earth history [3], associated with major evolutionary innovations and environmental upheavals [11]. 
How can such regulation arise? How does it persist? And: Why is the environment currently regulated in a regime that permits complex life? Answering these questions is important for several reasons: Firstly, to address scientifically how humanity came to be here [11] and the probability of detecting life elsewhere [12]. Secondly, as we enter the Anthropocene, it can offer fundamental insights about how resilient (or not) the Earth system is to anthropogenic perturbation [13]. Thirdly, it can elucidate mechanisms important for generating stability and persistence in other scales of life-environment system.

Most studies of Gaia presume that global-scale self-regulation involving life must arise through some form of natural selection $[4,14,15]$, and then raise several problems, notably: Selection amongst a population of interacting biospheres is not plausible, and natural selection for environmental regulation at smaller scales seems to imply a cooperative solution that would be vulnerable to 'cheats' [16-19]. However, stability is a general property of dynamical systems, which can be explicable in terms of feedback principles and self-organisation [4, 20, 21]. Whilst natural selection operating at conventional scales can, in special cases, contribute to environmental regulation [4, 22, 23], recent work has shown that regulatory outcomes can be selected by alternative mechanisms acting across a range of spatial and temporal scales [14, 18, 22, 24-28] (setting aside whether these are just forms of natural selection [28-30]).

Here we synthesize these selection mechanisms to show how environmental regulation can arise across multiple scales (Figure 1). Both new theory $[14,18]$ and recent advances in characterising microbial community evolution and effects on biogeochemical cycling [31, 32] make this timely. We start by addressing the role of natural selection in environmental 
regulation, then turn to how global long-timescale regulation can arise, before considering the role of anthropic (observer) bias.

\section{Natural selection and environmental regulation}

Daisyworld [23] provided a hypothetical demonstration that biological environmental feedbacks could in principle give rise to self-regulation at global scale. The coupling to the environment is via population dynamics: when it is cold, low-albedo black daisies are selected and their spread warms up the planet, but when it is hot the high-albedo white daisies are selected and their spread cools the planet. As a result, Daisyworld stabilises in a 'rein control' regime [33] which opposes perturbations in either (warmer or cooler) direction [34].

Although Daisyworld was presented as a "parable" [23], the model is so elegant, and so many studies have followed up on it, that it might have created a false impression of the likely nature of global regulatory mechanisms and their relationship with individual-based natural selection [35]. Daisyworld is a special case in that traits selected at an individual scale also lead to global regulation. The micro-evolutionary dynamics are therefore stabilising, addressing the persistence of regulation and illustrating a key feature of any plausible regulation mechanism - but providing no explanation for how or why a biota with these properties would arise. Nevertheless, the relationship in Daisyworld between population dynamics and environmental regulation mediated by 'leaky' ecological public goods is very similar to the 'Black Queen' hypothesis [36-39] (see Glossary), or the marine nitrogen cycle (Table 1) in which nitrogen fixers gain a private benefit and create a (costly) public good [39], giving them negative frequency dependent fitness that leads to regulation of available nitrogen levels [5-8]. 
81 To address how regulation could arise, some recent models $[22,25,26,40]$ (Box 1 ) have sought to consistently model Darwinian evolutionary processes within local environments which can then aggregate to form a larger-scale (potentially global) environment.

\section{Evolution of recycling loops}

An important question for the early evolution of any biosphere, is how readily (or not) the recycling of bio-essential materials (out of which organisms build their bodies) can be established, and by what mechanisms [9]. Recycling was integral to the origin of life [41] and subsequently, efficient and persistent nutrient recycling was essential for life to have a substantial effect on the abiotic environment. The surface of the Earth is close to being a materially closed system; i.e. the exchange fluxes of materials between the surface where life resides and the solid Earth or outer space are generally meagre [42]. Consider the gaseous exchange fluxes of materials between the Earth's surface and atmosphere (Figure 2): The fluxes coming from volcanic and metamorphic processes today are tiny compared to those due to the contemporary biosphere. The problem would have been even more acute prior to the establishment of plate tectonics as a slow recycling mechanism for volatiles which some studies place long after the start of life on Earth [43]. Equally when planets (e.g. Mars) are not materially closed to outer space and lose hydrogen too readily, it can pose a serious habitability problem because they lose water.

Establishment of closed recycling loops robustly occurs in the 'Flask' model (Box 1) of a 'microbial' community in which new metabolic pathways can stochastically emerge [22]. This is explained by conventional natural selection: The waste by-products of an organism's metabolism represent a potential resource and metabolic innovations that make use of that resource as a substrate for growth will be selected if the benefit outweighs the cost. Once a 
recycling loop is closed (such that every substrate is both consumed and produced), the closure of that loop increases the growth and replication rates (i.e. fitness) of all its constituent members in a positive feedback process. Co-production of by-products can thus be subject to positive selection (e.g. syntrophy) [15]. Members of the recycling loop are no longer limited by the external supply of essential materials into their (localised) environment, but rather by the efficiency with which they can recycle them. A closed recycling loop implies an external source of free energy, typically entering via photoautotrophs, even though the remaining steps in a recycling loop are often exothermic (energy yielding) [44].

Recycling systems are micro-evolutionarily stable, but can be disrupted if there are underutilised resources in the environment and a new trait arises which can utilise them [22] (Box 1) - for example, humans accessing fossil fuels [45].

\section{Multi-level selection}

Clearly not all aspects of the environment are resources for organisms, but physical qualities such as temperature and $\mathrm{pH}$ can nevertheless constrain the growth and replication of organisms, raising the question of whether and how these (non-resource) environmental variables can be regulated.

Empirical studies demonstrate that artificial ecosystem selection for environmental 'traits' can be effective [46], and both empirical [46] and modelling [47] work suggests this is not just because it implicitly selects for a single species that controls the chosen environmental effect. Thus, if there is some heritable variation in the environmental effects of communities (e.g. derived from genetically-based interactions within and between species [48]), which affect the growth of their members, leading to their differential replication or spread, 
ecosystem-level natural selection for environmental traits could occur. It requires some spatial structure, which can be a pre-existing feature of the environment [26] or be selfgenerated by communities [49].

A spatial extension of the 'Flask' model [26] (Box 1) illustrates how multi-level selection for environmental regulation could occur (Figure 3). Assuming a meta-community structure [50], communities whose aggregate effects on their locally-shared environment improve (individual) growth - and hence population density - spread at the expense of communities that degrade their local environment and hence are less densely populated. Consequently, the global environment becomes, on average, better regulated over time. The selection mechanism relies on some (imperfect) heritability of ecosystems, in which their key metabolic components can recombine to form the same ecosystem functional unit in neighbouring locations, even though their transfer through space is piecemeal in time (modelled as neutral dispersal by mixing). In reality there is growing evidence for microbial community coalescence (coherent dispersal) [51] and when communities are mixed the most metabolically efficient comes to dominate $[52,53]$.

Higher-level selection can also overcome a net cost to closing a recycling loop that is vulnerable to 'cheating', because mutualistic nutrient recycling communities have greater population density and spread at the expense of communities dominated by cheats [54].

However, this mechanism only works for spatially heterogeneous environmental variables where the effects on growth are contemporaneous with the organisms that cause them. It does not deal with globally well-mixed variables or address long time delays that can disable negative feedback and promote instability [55]. Some key Earth system variables are well 


\section{Sequential selection for stability}

152 A purely dynamical mechanism to favour stability is simply that "fragile systems are fleeting" [56] whereas stable regimes are attractors in phase space, hence a system spends longer 154 there.

mixed with long residence times (Table 1), therefore the world is a single 'flask' and a different mechanism of generating regulation is needed [40].

An early example was the concept of 'ultrastability' - or "reconfigure when things go wrong" - introduced by cybernetics pioneer W. Ross Ashby in the late 1940s $[57,58]$ to try and explain neurophysiological self-regulation [58]. Ashby illustrated the general mechanism with a machine called the 'homeostat' which he built out of four World War II bomb navigation devices. The system controlled four 'essential' variables, with a feedback configuration that randomly rewired its connections when the essential variables departed from a prescribed range, doing so repeatedly until it 'found' a stable regime. External forcing could knock the system out of a stable attractor, leading to a repeat of the random search for a new stable attractor.

To apply this principle of dynamical self-organisation to the regulation of the biosphere (with globally well-mixed, 'slow' variables) we introduced the notion of sequential selection [59-61], which was partly inspired by correspondence with the late W. D. Hamilton: "I imagine that "learning" through repetitions over time alone in a sufficiently complex system has to be shown able to replace the currently understood (and I am sure much more powerful) "learning" through repetitions over both time and space that is natural selection as we know it" [62]. 
171 To illustrate how sequential selection for stability might work for the Earth we summarise it

172 as an algorithm (Figure 4) and consider the extreme case where the events that separate

173 the repetitions in sequence are near-fatal 'resets' of the global system $[61,62]$. The

174 algorithm starts when life originates somehow. Subsequent evolutionary innovations will

175 inevitably lead to environmental effects [63]. These could improve or degrade the environment for the growth of the incumbent biota. Environment-improving innovations

177 produce positive feedback on growth, which if strong enough can pass through optimum conditions and cause a switch to environment-degrading effects creating negative feedback on growth and convergence on a stable attractor. The Gaia puzzle is in this case 'solved' (at least temporarily) without the need for repetition. However, if the initial effects are environment-degrading and there is timescale separation such that negative feedback on growth does not kick in quickly, or if some abiotic positive feedback is triggered, then the system might approach the bounds of habitability. This could for example take the form of a 'snowball Earth' event [64] in which the planet is nearly completely frozen over and pockets of life are left isolated in small 'oases' (e.g. geothermal settings). Approaching the bounds of habitability effectively resets the system allowing a new repetition to unfold. Again the effects of life on the environment might be beneficial to current life forms, or not, and if not the cycle can repeat itself. However, if the system arrives at a stable attractor it will by definition persist until destabilised by a further evolutionary innovation (or external forcing).

Several recent models illustrate forms of sequential selection for environmental regulation (Box 2) $[22,24,25,27,65,66]$, building on earlier examples of sequential selection in models of community assembly [19, 67-69]. All involve the same fundamental principles, where a set of components (to some extent predetermined by the model structure) self- 
organize into stable configurations that regulate their environment, through single feedbacks or rein control. They all rely on some separation of (fast) ecological and (slow) evolutionary timescales, including; population dynamics - the system regulates or does not; micro-evolutionary dynamics - the population adapts or does not, and macro-evolution new types of life arise or are introduced. Stability can be disrupted either by mutation, community assembly, or external forcing, and can be re-found by sequential selection. Overall, trends towards increased stability can occur [66].

One of the models [65], shows that the number of potential stable attractors increases exponentially with the number of environmental variables, but the likelihood of the system being in a stable attractor within prescribed habitability bounds declines approximately linearly. This mirrors the behaviour of Ashby's homeostat in which increasing the number of homeostat units increases the amount of time required for the system to establish a regulating state. Overall, these studies show that sequential selection is satisficing rather than optimising, because unlike natural selection it cannot refine regulatory mechanisms over time.

\section{Selection by survival}

An even simpler mechanism than sequential selection, of selection based on survival (persistence) alone $[30,70]$, could help explain the acquisition of regulatory mechanisms at the planetary scale [18]. In essence; persistence increases the likelihood of acquiring further persistence-enhancing traits [18]. Specifically, differential survival of non-competing and non-reproducing individuals, which exhibit variation, will result in increasing frequencies of survival-promoting "adaptations" among survivors [18]. This mechanism can operate even in a population of one Earth, and the persistence-enhancing traits could include planetary- 
scale homeostatic mechanisms. But unlike sequential selection, the acquisition of

homeostatic mechanisms has been portrayed as a stochastic process [18] - there is no cybernetic or algorithmic conception of a search through a complex phase space that 'finds' homeostatic attractors [56]. Instead we suggest that homeostatic mechanisms found by sequential selection (Figures 4,5 ) could be accumulated or improved through selection by survival.

Recent work [14] has considered "the biogeochemical cycles and other homeostatic processes that might confer stability - rather than the taxa (mostly microbial) that implement them - as the relevant units of selection". This allows for a population of interacting (albeit non-reproducing) entities subject to selection based on survival - in this case the differential persistence of different variants of a biogeochemical cycle [14].

Conceptually, given the nearly materially-closed nature of the system (Figure 2), there could indeed be more than one form of e.g. nitrogen cycle, 'competing' to cycle a finite amount of biologically-available nitrogen. Furthermore, variations between realisations of a cycle could conceivably be traced down to underlying differences in key genes that are each shared across many phyla and exchanged laterally [44, 71, 72]. A leap of scale might therefore be made between the large-scale functional biogeochemical manifestations of metabolisms and the small-scale of the genes encoding for those metabolisms, by-passing the individuals, species and taxonomic composition between [14, 72-74].

At smaller scales, selection based on non-heritable variation but differential persistence of ecosystems provides an alternative mechanism for ecosystem evolution $[30,67,70,75]$ to one based on heritable variation [26] (discussed above). 
239

240

241

242

243

244

245

246

247

248

249

250

251

252

253

254

255

256

257

258

259

\section{Progress by accumulation}

Having considered why the Earth has self-stabilising properties, a natural follow-on question

is; why does it appear to show progressive development of certain properties? Specifically, the productivity of the biosphere [45] and the level of oxygen in the atmosphere [42] have both increased in a series of steps. Combining natural selection, sequential selection and selection by survival suggests that directional trends should emerge at the macro-scale. Natural selection should tend to give rise to increasing primary productivity because any individual that is able to capture more free energy than its compatriots, e.g. through an improved form of photosynthesis, can grow faster and produce more descendants [63]. Improvements in recycling cause step-increases in biosphere productivity, as the 'Flask' model shows (Box 1) $[22,25]$. Furthermore, under-exploited resources will eventually be tapped - and the resulting rapid growth can kick the system into a new configuration [25]. More productive systems tend to be more stable by virtue of having already exploited potentially disruptive resource stocks $[25,66]$. All this can help explain the stepwise oxygen rise over Earth history, because oxygen is the waste by-product of the most effective biological means of free-energy capture - oxygenic photosynthesis. Indeed recent modelling of long-term oxygen regulation has reopened the possibility that an increase in the productivity of the early oxygenic photosynthetic biosphere could have triggered the Great

Oxidation ( 2.45-2.3 Ga) [76]. Furthermore, the approximate doubling of global primary productivity that accompanied the rise of land plants ( $400 \mathrm{Ma})$ triggered a second oxygenation event that finally brought atmospheric $\mathrm{O}_{2}$ to modern levels [77]. 
260

261

262

263

264

265

266

267

268

269

270

271

272

273

274

276

277

\section{Observer self-selection}

On the largest space and longest time scales observer self-selection also needs to be considered: The history of Earth that we see has to be consistent with our existence as conscious observers [11, 78-81]. For example, Earth history had to include a rise in atmospheric oxygen to near present levels sufficient to support $20 \mathrm{~W}$ brain function [11, 82], for us to be here to wonder about it. This is a form of the weak anthropic principle [83].

Extending this principle to environmental regulation, a biosphere able to support complex self-aware life likely requires a certain number and/or strength of stabilising feedbacks.

Whilst the sequential selection mechanism can discover stable states, at least in its simplest form it does not require the existence of any particular biosphere (or any biosphere at all, if we relax the condition of stabilising in a habitable state) (Figure 5). The majority of Earthlike rocky planets might therefore stabilise in regimes very different from modern Earth [84]. Our existence also requires directionality, which could in part be an observer bias and not an intrinsic property (implying that biospheres on average would show no such trend). Taken together, this includes biosphere regulation $[81,85]$ in Carter's original deduction [86] that our relatively late evolution in the lifespan of the biosphere implies a small number of highly improbable steps in the evolution of complex life.

\section{Testing the theory}

The 'why stability?' question has framed much of the debate about Gaia and related modelling work thus far, but we argue that it is now partly answered, in theory at least. However, there remain many outstanding questions (Box 3) and much scope for empirical testing of the proposed mechanisms: 
282

283

284

285

286

287

\section{Earth history}

Earth history provides examples of regulatory regimes, transitions between them, and external perturbations [11]. Only the modern Phanerozoic regime is currently understood in any detail [87]. Better understanding of the Archean [88] and Proterozoic [76] regimes and the transitions between regimes $[76,77,88,89]$ could shed light on how the sequential selection mechanism operates: It might not require pushing the bounds of habitability. Similarly external perturbations [56] might result in secular increases in biosphere stability (Figure 5). It is unclear how much relevant global scale 'memory' can be carried in the pangenome [90] and phylogeny of life, or the biosphere organisation itself [91, 92]. Earth history can also test observer self-selection models [11, 81].

\section{Experimental systems}

Real microbial microcosms [15] in which species are randomly added (community assembly) or experimental evolution occurs could be designed to test predictions regarding the likelihood of occurrence and robustness of nutrient recycling [22], and the mechanism for regulation of heterogeneous environmental variables [26]. This could build on recent studies of artificial ecosystem selection [46], the Black Queen hypothesis [37, 38], and community coalescence $[52,53]$. Already alternative stable states in real nutrient recycling microbial ecosystems have been identified [93], qualitatively consistent with models [40]. Larger scale ecosystem construction experiments could follow in the spirit of the Ecotron experiments [94], but testing the potential for systems to regulate environmental variables - noting the salutary lessons from 'Biosphere 2' [95]. 
303

304

\section{Extrasolar planets}

Exoplanets estimated to be in the 'habitable zone' of their parent star (with liquid water at their surface) have recently been discovered [96-99] and at least one is expected around roughly every fifth star [99]. The technology to detect abundant life on these planets through its effect on atmospheric composition [100], is being developed [99]. Hence in the coming decades we can hope to find out whether potentially habitable extrasolar planets are inhabited [99]. Aiming for a large sample size [101] could help test the Gaia hypothesis [12] and observer self-selection [81]: If there is a propensity for stabilising life-planet outcomes, then this should shift the age distribution of inhabitation through the lifetime of potentially habitable planets older than if there is anti-Gaia (younger-skewed) or neutral effects [85]. The challenge is establishing the null expectation to test against.

\section{Concluding remarks}

The taxonomy of mechanisms we have discussed spans variational and transformational evolution [102]. Natural selection can produce nutrient (re)cycling including the restricted production and regulation of 'leaky' ecological public goods subject to negative frequencydependent selection. Microbial ecosystem-level selection could give rise to regulation of heterogeneous environmental variables based on the differential spread (with heritability aided by community coalescence) or persistence of environment-improving ecosystems. However, regulation of environmental variables at global scales and on long timescales requires a different explanation. Building on existing work $[15,69,78,103]$, we introduce a new constraint - a dynamical filtering for stability consistent with Earth system feedbacks between the origin of major evolutionary innovations and their persistence in the biosphere: Sequential selection of stable configurations enhancing the persistence of the 
326

327

328

329

330

332

biosphere can in turn increase the likelihood of acquiring further persistence-enhancing properties through selection based on survival alone. The outstanding challenge is to establish empirically the effectiveness and importance of these different mechanisms.

\section{Acknowledgments}

We thank Richard Betts, Richard Boyle, Inman Harvey, Jim Lovelock and Andy Watson for helping shape our ideas, and four anonymous referees for their generous feedback. T.M.L. was supported by a Royal Society Wolfson Research Merit Award. A.E.N. was supported by Gaia Charity and the University of Exeter. 
335

336

337

\section{Box 1: Variants of the Flask model}

The original single-Flask model [22] simulates a well-mixed container of fluid, with interactive nutrient levels and (non-nutrient) environmental variables (e.g. temperature). Nutrient input fluxes and corresponding fluid outflow are prescribed. The flask is seeded with a clonal population of individual 'microbes' each containing a 'genome' that determines their phenotypic traits of nutrient uptake, release, (by-product) effects on, and response to environmental variables. Microbes grow dependent on their nutrient uptake and the state of the environment and replicate (asexually) above a threshold size. Random mutation generates genetic variation and natural selection occurs. Unlike Daisyworld [23], what is selected at the individual level is decoupled from its environmental effects.

Ecosystems emerge that tend toward a state where nutrients are efficiently utilized and differentially recycled, increasing total population size [22]. When microbes have no shared environmental preference and no constraints on the conditions to which they can adapt, 'rebel' organisms can appear that grow rapidly by exploiting an under-utilized resource, but shift the environment away from the state to which the majority of the community are adapted, causing population crashes followed by recovery, or in extreme cases, total extinction of the system. When microbes are given a shared, fixed environmental preference then environmental regulation can arise either above or below the optimum for growth [40]. When new mutants arise that alter the magnitude of the net environmental effect of the community, the total population expands or contracts to counter this, but mutants that change the sign of the environmental effect can cause switches between regulatory regimes [40]. 
357 In a spatially-extended version of the model [26] a series of (individually homogeneous)

358 'flasks' are connected together with imperfect mixing between them to create a

359 heterogeneous collective environment. When all organisms are given the same growth

360 response to the environment (but are genetically different in their nutrient requirements),

361 there is ecosystem-level selection for environmental regulation (Figure 3). If closing a

362 recycling loop is assumed to carry a fitness cost, then ecosystem-level selection can counter

363 individual-selection and allow nutrient recycling to spread at the expense of cheating [54].

364 When the growth response of microbes to the environment is allowed to adapt, transitions

365 between regulatory regimes can occur [25]. 'Rebel' organisms again cause abrupt

366 environmental changes that drive incumbent species extinct, resulting in ecosystem collapse

367 followed by recovery to a new stable state, or occasionally system-wide extinction.

368 However, ensemble-level results show increasing mean ecosystem productivity and stability

369 over time as resources are progressively exploited. 
372

\section{Box 2: Models illustrating sequential selection}

\section{Greenhouse world}

In 'Greenhouse world' [27] organisms affect a well-mixed atmosphere and thus global temperature, they have different growth responses to temperature that can adapt (without physiological limits), and occasionally new members are randomly added to the community.

This separates ecological (population dynamics), micro-evolutionary, and macroevolutionary (community assembly) timescales. Randomly generated communities typically display winnowing phases of sequential selection in which unstable environmental variation leads to one or more species extinctions, until a simpler, stable configuration is found. Introducing a new species can disrupt a stable community leading to a repeat of the sequential selection search for a stable configuration. Stability is typically (re)found 10-30 times before a global extinction event - which can occur when an invading species triggers a runaway positive feedback process of extinctions and escalating environmental change.

\section{Daisystat}

In the 'daisystat' model [24] organisms affect a well-mixed global environment through byproducts of their metabolism, organisms can only survive in a certain environment (i.e. niche), and the rate of evolutionary adaptation is assumed slower than the rate at which niche construction activities can change the environment (separating ecological and microevolution timescales). The population contains environment increasing and decreasing alleles and a range of peaked growth responses with different, adapting environmental optima. The system transits rapidly through positive feedback regimes in which e.g. the organisms closest to their optimum increase the environmental variable and then the next growth response selected for also drives it up, before finding and stabilising in 'rein control' 
regimes [33], when e.g. encountering organisms that decrease the environmental variable despite having a higher preference for it. Environmental forcing and/or genetic drift can cause the exit from a stable attractor and sequential selection for a new stable attractor.

\section{$398 \quad$ Tangled Nature}

An extension of the 'Tangled Nature Model' [66] allows agents to affect the carrying capacity of their world. Emerging species can cause collapses or rearrangements of quasistable states that include a core of dominant species. A collapse creates a vacuum, which is likely to be filled by species with high growth rates and leads to a gradually increasing total population. Because of resource competition with larger populations, new mutants find it

404 more difficult to become established, leading to fewer ecosystem collapses with time. Sequential selection occurs because environment-degrading ecosystems have smaller populations and are more prone to collapse compared to environment-improving ecosystems. The result is that environment-improving adaptations are favoured over time and/or ensemble average. 


\section{Box 3: Outstanding questions}

- Magnitude of effects: What impact do the different mechanisms for selecting environmental regulatory outcomes have on the probability of life persisting on a planet? Have they played a significant role on Earth?

- Relative importance of mechanisms: What is the relative contribution of natural selection, sequential selection and selection by survival to the creation of environmental regulation observed at different spatial and temporal scales?

- Evolutionary ecology: For what variables and over what scale(s) can the Black Queen Hypothesis help explain environmental regulation? Can community coalescence provide heritability of microbial ecosystems?

- Ecosystem selection: Does ecosystem-level natural selection and/or selection-bysurvival of ecosystems occur? How important is either for environmental regulation?

- Identifying a Gaia "lab rat": Could a real microcosm be built that captures the essential properties and constraints of the Earth system? If so, which postulated mechanisms for selecting environmental regulatory outcomes could it test?

- Role of (bio)diversity in stability: What is the role of biodiversity in establishing and maintaining stable states? Do diverse populations allow greater resilience to perturbation?

- Anthropocene: How stable is the Earth system to human perturbation? How readily can environmental regulation mechanisms be regenerated if human activities disrupt or eliminate them?

- Implications of inhabitation for habitability: Is the present biosphere maintaining a habitable state on Earth when otherwise it would be(come) uninhabitable? How is the notion of a 'habitable zone' altered by life? 
434

435

436

437

438

439

440

- Testing Gaia with exoplanets: What is the null hypothesis for the distribution of inhabitation of potentially habitable planets as a function of time through star and planet lifetime? What sample size of inhabited planets would be required to test the Gaia hypothesis and/or observer self-selection against the null? What might evidence of a 'failed Gaia' look like? 
441 Glossary

442 Attractor: A set of numerical values toward which a system tends to evolve, for a wide

443 variety of starting conditions of the system, and to which it tends to return for some range

444 of perturbations.

445 Biotic plunder: Biological populations tend to proliferate when conditions are favourable,

446 drawing down resources to limiting levels, which tend to stay that way.

447 Black Queen hypothesis: Loss of a costly, leaky function is selectively favored at the

448 individual level and will proceed until the production of public goods is just sufficient to

449 support the equilibrium community.

450 By-product: A consequence of a phenotype selected for other reasons, e.g. environmental

451 changes due to excreted metabolic waste products. By-products can subsequently become

452 selected for e.g. if they form the basis of a closed recycling loop.

453 Ecosystem evolution: Either selection by survival operating on variation in ecosystem-level

454 properties, or natural selection operating on heritable variation in ecosystem-level

455 properties.

456 Macroevolution: evolution on a scale at or above the level of species (i.e. operating on

457 separated gene pools), e.g. community assembly, clade selection.

458 Microevolution: small evolutionary changes within a species or population (i.e. operating

459 within a gene pool).

460 Natural selection: Heritable variation in phenotypic traits among members of a population

461 results in increasing frequencies of descendant-producing properties among descendants. 
462 Observer self-selection: An application of the weak anthropic principle: The nature of the

463 universe including the history of the Earth has to be consistent with our existence as

464 conscious observers.

465 Rein control: Stabilising control of a particular variable (here environmental) by two

466 feedbacks which individually pull that variable in opposite directions.

467 Selection by survival: Differential survival of non-reproducing (and in some cases non-

468 competing) entities, which exhibit variation, results in increasing frequencies of survival-

469 promoting properties among survivors.

470 Sequential selection: Repetitions of a system over time alone enable it to acquire stabilising

471 mechanisms because fragile systems are fleeting whereas stable configurations tend to

472 persist.

473 Snowball Earth hypothesis: The proposition that the Earth's surface became (nearly)

474 entirely frozen for a geological period of time, several times during the Precambrian.

475 Ultrastability: The property of a system able to change its internal structure in response to

476 perturbations that affect some "essential variables", which if driven outside of particular

477 values produce a step-change reconfiguration of feedbacks until a new stable state is found. 
479

480

481

482

483

484

485

486

487

488

489

490

491

492

493

494

495

496

497

498

499

500

501

\section{References}

1. Lovelock, J.E. (1972) Gaia as seen through the atmosphere. Atmospheric Environment 6, 579-580

2. Lovelock, J.E. (1979) Gaia - A New Look at Life on Earth. Oxford University Press

3. Lovelock, J.E. (1988) The Ages of Gaia - A Biography of Our Living Earth. W. W. Norton \& Co.

4. Lenton, T.M. (1998) Gaia and natural selection. Nature 394, 439-447

5. Redfield, A.C. (1958) The biological control of chemical factors in the environment. American Scientist 46, 205-221

6. Lenton, T.M., and Watson, A.J. (2000) Redfield revisited: 1. Regulation of nitrate, phosphate and oxygen in the ocean. Global Biogeochemical Cycles 14, 225-248

7. Auguères, A.-S., and Loreau, M. (2015) Regulation of Redfield ratios in the deep ocean. Global Biogeochemical Cycles 29, 254-266

8. Tyrrell, T. (1999) The relative influences of nitrogen and phosphorus on oceanic primary production. Nature $400,525-531$

9. Volk, T. (1998) Gaia's Body - Toward a Physiology of the Earth. Copernicus

10. Auguères, A.-S., and Loreau, M. (2015) Can Organisms Regulate Global Biogeochemical Cycles? Ecosystems 18, 813-825

11. Lenton, T.M., and Watson, A.J. (2011) Revolutions that made the Earth. Oxford University Press

12. Nicholson, A.E., et al. (2018) Gaian bottlenecks and planetary habitability maintained by evolving model biospheres: The ExoGaia model. Monthly Notices of the Royal

\section{Astronomical Society, sty658}


502

503

504

505

506

507

508

509

510

511

512

513

514

515

516

517

518

519

520

521

522

523

524

13. Steffen, W., et al. (2015) Planetary boundaries: Guiding human development on a changing planet. Science 347,1259855

14. Doolittle, W.F. (2017) Darwinizing Gaia. Journal of Theoretical Biology 434, 11-19

15. Free, A., and Barton, N.H. (2007) Do evolution and ecology need the Gaia

hypothesis? Trends in Ecology \& Evolution 22, 611-619

16. Doolittle, W.F. (1981) Is Nature Really Motherly? The CoEvolution Quarterly Spring, $58-63$

17. Dawkins, R. (1983) The Extended Phenotype. Oxford University Press

18. Doolittle, W.F. (2014) Natural selection through survival alone, and the possibility of Gaia. Biology \& Philosophy 29, 415-423

19. Hamilton, W.D. (1995) Ecology in the Large: Gaia and Genghis Khan. Journal of Applied Ecology 32, 451-453

20. Levin, S.A. (1998) Ecosystems and the Biosphere as Complex Adaptive Systems. Ecosystems 1, 431-436

21. Levin, S.A. (2005) Self-organization and the Emergence of Complexity in Ecological Systems. BioScience 55, 1075-1079

22. Williams, H.T.P., and Lenton, T.M. (2007) The Flask model: Emergence of nutrientrecycling microbial ecosystems and their disruption by environment-altering 'rebel' organisms. Oikos 116, 1087-1105

23. Watson, A.J., and Lovelock, J.E. (1983) Biological homeostasis of the global environment: the parable of Daisyworld. Tellus 35B, 284-289

24. McDonald-Gibson, J., et al. (2008) Environmental regulation can arise under minimal assumptions. Journal of Theoretical Biology 251, 653-666 
25. Williams, H.T.P., and Lenton, T.M. (2010) Evolutionary regime shifts in simulated ecosystems. Oikos 119, 1887-1899

26. Williams, H.T.P., and Lenton, T.M. (2008) Environmental regulation in a network of simulated microbial ecosystems. Proceedings of the National Academy of Sciences 105, 10432-10437

27. Worden, L. (2010) Notes from the greenhouse world: A study in coevolution, planetary sustainability, and community structure. Ecological Economics 69, 762-769 28. Toman, J., and Flegr, J. (2017) Stability-based sorting: The forgotten process behind (not only) biological evolution. Journal of Theoretical Biology 435, 29-41

29. Bourrat, P. (2014) From survivors to replicators: evolution by natural selection revisited. Biology \& Philosophy 29, 517-538

30. Bouchard, F. (2014) Ecosystem Evolution is About Variation and Persistence, not Populations and Reproduction. Biological Theory 9, 382-391

31. Mock, T., et al. (2016) Bridging the gap between omics and earth system science to better understand how environmental change impacts marine microbes. Global Change Biology 22, 61-75

32. Stolz, J.F. (2017) Gaia and her microbiome. FEMS Microbiology Ecology 93, fiw247

33. Clynes, M. (1969) Cybernetic implications of rein control in perceptual and conceptual organization. Annals of the New York Academy of Sciences 156, 629-664

34. Saunders, P.T., et al. (1998) Integral Rein Control in Physiology. Journal of theoretical Biology 194, 163-173

35. Wood, A.J., et al. (2008) Daisyworld: A review. Reviews of Geophysics 46, RG1001 36. Morris, J.J., et al. (2012) The Black Queen Hypothesis: Evolution of Dependencies through Adaptive Gene Loss. mBio 3, e00036-00012 
549

550

551

552

553

554

555

556

557

558

559

560

561

562

563

564

565

566

567

568

569

570

571

37. Morris, J.J., et al. (2014) COEXISTENCE OF EVOLVING BACTERIA STABILIZED BY A

SHARED BLACK QUEEN FUNCTION. Evolution 68, 2960-2971

38. Estrela, S., et al. (2016) Private benefits and metabolic conflicts shape the emergence of microbial interdependencies. Environmental Microbiology 18, 1415-1427

39. Morris, J.J. (2015) Black Queen evolution: the role of leakiness in structuring microbial communities. Trends in Genetics 31, 475-482

40. Nicholson, A.E., et al. (2017) Multiple states of environmental regulation in wellmixed model biospheres. Journal of Theoretical Biology 414, 17-34

41. King, G.A.M. (1982) Recycling, reproduction, and life's origins. Biosystems 15, 89-97

42. Lenton, T. (2016) Earth System Science: A Very Short Introduction. Oxford University Press

43. Korenaga, J. (2013) Initiation and Evolution of Plate Tectonics on Earth: Theories and Observations. Annual Review of Earth and Planetary Sciences 41, 117-151

44. Jelen, B.I., et al. (2016) The Role of Microbial Electron Transfer in the Coevolution of the Biosphere and Geosphere. Annual Review of Microbiology 70, 45-62

45. Lenton, T.M., et al. (2016) Revolutions in energy input and material cycling in Earth history and human history. Earth Syst. Dynam. 7, 353-370

46. Blouin, M., et al. (2015) Levels and limits in artificial selection of communities.

Ecology Letters 18, 1040-1048

47. Williams, H.T.P., and Lenton, T.M. (2007) Artificial selection of simulated microbial ecosystems. Proceedings of the National Academy of Sciences 104, 8918-8923

48. Goodnight, C.J. (2000) Heritability at the ecosystem level. Proceedings of the National Academy of Sciences 97, 9365-9366 
572

573

574

575

576

577

578

579

580

581

582

583

584

585

586

587

588

589

590

591

592

593

594

595

49. Johnson, C.R., and Boerlijst, M.C. (2002) Selection at the level of the community: the importance of spatial structure. Trends in Ecology \& Evolution 17, 83-90

50. Leibold, M.A., et al. (2004) The metacommunity concept: a framework for multiscale community ecology. Ecology Letters 7, 601-613

51. Rillig, M.C., et al. (2015) Interchange of entire communities: microbial community coalescence. Trends in Ecology \& Evolution 30, 470-476

52. Sierocinski, P., et al. (2017) A Single Community Dominates Structure and Function of a Mixture of Multiple Methanogenic Communities. Current Biology 27, 3390-3395.e3394

53. Rillig, M.C., and Mansour, I. (2017) Microbial Ecology: Community Coalescence Stirs Things Up. Current Biology 27, R1280-R1282

54. Boyle, R.A., et al. (2012) Natural selection for costly nutrient recycling in simulated microbial metacommunities. Journal of Theoretical Biology 312, 1-12

55. Weaver, I.S., and Dyke, J.G. (2012) The importance of timescales for the emergence of environmental self-regulation. Journal of Theoretical Biology 313, 172-180

56. Wagner, A. (2007) Robustness and Evolvability in Living Systems. Princeton University Press

57. Ashby, W.R. (1948) Design for a Brain. Electronic Engineering 20, 379-383

58. Ashby, W.R. (1952) Design for a Brain. Chapman \& Hall

59. Lenton, T.M. (2004) Clarifying Gaia: Regulation with or without Natural Selection. In

Scientists Debate Gaia: The Next Century (Schneider, S.H., et al., eds), 15-25, MIT Press

60. Lenton, T.M., et al. (2004) What does history teach us about the major transitions and the role of disturbances in the evolution of life and of the Earth system? In Earth System Analysis for Sustainability, Dahlem Workshop Report 91 (Schellnhuber, H.-J., et al., eds), 2952, The MIT Press 
596

597

598

599

600

601

602

603

604

605

606

607

608

609

610

611

612

613

614

615

616

617

61. Betts, R.A., and Lenton, T.M. (2007) Second chances for Lucky Gaia: a hypothesis of sequential selection. In Gaia Circular, 4-6

62. Lenton, T.M. (2005) Hamilton and Gaia. In Narrow Roads of Gene Land - The Collected Papers of W. D. Hamilton. Volume 3 - Last Words (Ridley, M., ed), 257-264, Oxford University Press

63. Schrödinger, E. (1944) What is Life? Cambridge University Press

64. Hoffman, P.F., and Schrag, D.P. (2002) The snowball Earth hypothesis: testing the limits of global change. Terra Nova 14, 129-155

65. Dyke, J.G., and Weaver, I.S. (2013) The Emergence of Environmental Homeostasis in Complex Ecosystems. PLOS Computational Biology 9, e1003050

66. Arthur, R., and Nicholson, A. (2017) An entropic model of Gaia. Journal of Theoretical Biology 430, 177-184

67. Ott, J.A. (1981) Adaptive Strategies at the Ecosystem Level: Examples from Two Benthic Marine Systems. Marine Ecology 2, 113-158

68. Pimm, S.L. (1991) The Balance of Nature? The University of Chicago Press

69. Borrelli, J.J., et al. (2015) Selection on stability across ecological scales. Trends in Ecology \& Evolution 30, 417-425

70. Dussault, A.C., and Bouchard, F. (2017) A persistence enhancing propensity account of ecological function to explain ecosystem evolution. Synthese 194, 1115-1145

71. Falkowski, P.G., et al. (2008) The Microbial Engines That Drive Earth's

Biogeochemical Cycles. Science 320, 1034-1039

72. Burke, C., et al. (2011) Bacterial community assembly based on functional genes rather than species. Proceedings of the National Academy of Sciences 108, 14288-14293 
619 73. Louca, S., et al. (2016) Decoupling function and taxonomy in the global ocean 620 microbiome. Science 353, 1272-1277

621 74. Williams, G.R. (1996) The Molecular Biology of Gaia. Columbia University Press

622 75. Dunbar, M.J. (1960) The Evolution of Stability in Marine Environments Natural

623 Selection at the Level of the Ecosystem. The American Naturalist 94, 129-136

624 76. Daines, S.J., et al. (2017) Atmospheric oxygen regulation at low Proterozoic levels by 625 incomplete oxidative weathering of sedimentary organic carbon. Nature Communications 8, $626 \quad 14379$

627 77. Lenton, T.M., et al. (2016) Earliest land plants created modern levels of atmospheric 628 oxygen. Proceedings of the National Academy of Sciences 113, 9704-9709

78. Levin, S.A. (1999) Fragile Dominion: complexity and the commons. Perseus Books

79. Watson, A.J. (1999) Coevolution of the Earth's environment and life: Goldilocks, Gaia 631 and the anthropic principle. In James Hutton - Present and Future (Craig, G.Y., and Hull, J.H., eds), 75-88, Geological Society special publication

80. Watson, A.J. (2004) Gaia and observer self-selection. In Scientists Debate Gaia: The Next Century (Schneider, S.H., et al., eds), 201-208, MIT press

81. Watson, A.J. (2008) Implications of an Anthropic Model of Evolution for Emergence of Complex Life and Intelligence. Astrobiology 8, 175-185

82. Catling, D.C., et al. (2005) Why O2 Is Required by Complex Life on Habitable Planets and the Concept of Planetary "Oxygenation Time". Astrobiology 5, 415-438

83. Carter, B. (1974) Large Number Coincidences and the Anthropic Principle in

640 Cosmology. In Confrontation of Cosmological Theories with Observational Data (Longair, 
642

643

644

645

646

647

648

649

650

651

652

653

654

655

656

657

658

659

660

661

662

663

664

665

84. Lenardic, A., et al. (2016) The Solar System of Forking Paths: Bifurcations in Planetary

Evolution and the Search for Life-Bearing Planets in Our Galaxy. Astrobiology 16, 551-559

85. Chopra, A., and Lineweaver, C.H. (2016) The Case for a Gaian Bottleneck: The Biology of Habitability. Astrobiology 16, 7-22

86. Carter, B. (1983) The anthropic principle and its implications for biological evolution. Philosophical Transactions of the Royal Society A: Mathematical, Physical and Engineering Sciences 310, 347-363

87. Lenton, T.M., et al. (2018) COPSE reloaded: An improved model of biogeochemical cycling over Phanerozoic time. Earth Science Reviews 178, 1-28

88. Daines, S.J., and Lenton, T.M. (2016) The effect of widespread early aerobic marine ecosystems on methane cycling and the Great Oxidation. Earth and Planetary Science Letters 434, 42-51

89. Lenton, T.M., and Daines, S.J. (2017) Biogeochemical Transformations in the History of the Ocean. Annual Review of Marine Science 9, 31-58

90. Fullmer, M., et al. (2015) The pan-genome as a shared genomic resource: mutual cheating, cooperation and the black queen hypothesis. Frontiers in Microbiology 6

91. Watson, R.A., and Szathmáry, E. (2016) How Can Evolution Learn? Trends in Ecology \& Evolution $31,147-157$

92. Watson, R.A., et al. (2016) Evolutionary Connectionism: Algorithmic Principles Underlying the Evolution of Biological Organisation in Evo-Devo, Evo-Eco and Evolutionary Transitions. Evolutionary Biology 43, 553-581

93. Pagaling, E., et al. (2017) Assembly of microbial communities in replicate nutrientcycling model ecosystems follows divergent trajectories, leading to alternate stable states. Environmental Microbiology 19, 3374-3386 
666

667

668

669

670

671

672

673

674

675

676

677

678

679

680

681

682

683

684

685

686

687

94. Milcu, A., et al. (2012) Biotic carbon feedbacks in a materially closed soil-vegetationatmosphere system. Nature Climate Change 2, 281-284

95. Cohen, J.E., and Tilman, D. (1996) Biosphere 2 and Biodiversity--The Lessons So Far. Science $274,1150-1151$

96. Gillon, M., et al. (2017) Seven temperate terrestrial planets around the nearby ultracool dwarf star TRAPPIST-1. Nature 542, 456

97. Dittmann, J.A., et al. (2017) A temperate rocky super-Earth transiting a nearby cool star. Nature 544, 333

98. Anglada-Escudé, G., et al. (2016) A terrestrial planet candidate in a temperate orbit around Proxima Centauri. Nature 536, 437

99. Kaltenegger, L. (2017) How to Characterize Habitable Worlds and Signs of Life.

Annual Review of Astronomy and Astrophysics 55, 433-485

100. Lovelock, J.E. (1965) A physical basis for life detection experiments. Nature 207, 568570

101. Bean, J.L., et al. (2017) A Statistical Comparative Planetology Approach to the Hunt for Habitable Exoplanets and Life Beyond the Solar System. The Astrophysical Journal Letters 841, L24

102. Lewontin, R.C. (1983) The Organism as the Subject and Object of Evolution. Scientia $118,63-82$

103. Solé, R.V., et al. (2002) Self-organized instability in complex ecosystems.

Philosophical Transactions of the Royal Society of London. Series B: Biological Sciences 357, $667-681$ 
688 104. Tyrrell, T. (2004) Biotic Plunder: Control of the Environment by Biological Exhaustion 689 of Resources. In Scientists Debate Gaia: The Next Century (Schneider, S.H., et al., eds), 137-

690147, MIT press

691 105. Tilman, D. (1982) Resource Competition and Community Structure. Princeton

692 University Press

693 106. Lenton, T.M., and Watson, A.J. (2000) Redfield revisited: 2. What regulates the 694 oxygen content of the atmosphere? Global Biogeochemical Cycles 14, 249-268

695

696 
697

Table 1. Key regulated Earth system variables.

\begin{tabular}{|c|c|c|}
\hline Regulated variable & $\begin{array}{l}\text { Residence } \\
\text { time }\end{array}$ & Mechanism \\
\hline Ocean N & $10^{3}-10^{4} \mathrm{yr}$ & $\begin{array}{l}\text { 'Biotic plunder' [104] and resource } \\
\text { competition }\left(R^{*}\right)[105] \text { between } N \text { fixers and } \\
\text { non-fixers [6-8]. Decreased (increased) N } \\
\text { increases (decreases) N fixation. }\end{array}$ \\
\hline Ocean P & $10^{4}-10^{5} \mathrm{yr}$ & $\begin{array}{l}\text { 'Biotic plunder' [104]. Increased (decreased) } \\
\text { productivity increases (decreases) P removal } \\
{[6] .}\end{array}$ \\
\hline $\begin{array}{l}\text { Ocean-atmosphere } \mathrm{CO}_{2} \text { and } \\
\text { global temperature }\end{array}$ & $10^{5}-10^{6} \mathrm{yr}$ & $\begin{array}{l}\text { Silicate weathering with biotic enhancement } \\
\text { (local competition for nutrients) [87]. } \\
\text { Increased (decreased) } \mathrm{CO}_{2} \text { and temperature } \\
\text { increases (decreases) } \mathrm{CO}_{2} \text { removal. }\end{array}$ \\
\hline $\begin{array}{l}\text { Atmospheric } \mathrm{O}_{2} \text { (current } \\
\text { regulatory regime) }\end{array}$ & $10^{6}-10^{7} \mathrm{yr}$ & $\begin{array}{l}\text { Terrestrial biota overproduces } \mathrm{O}_{2} \text {, fire and/or } \\
\text { toxicity suppress } \mathrm{O}_{2} \text { production [106]. } \\
\text { Decreased } \mathrm{O}_{2} \text { increases terrestrial } \mathrm{O}_{2} \\
\text { production and ocean deoxygenation recycles } \\
\text { P increasing marine } \mathrm{O}_{2} \text { production [106]. }\end{array}$ \\
\hline
\end{tabular}




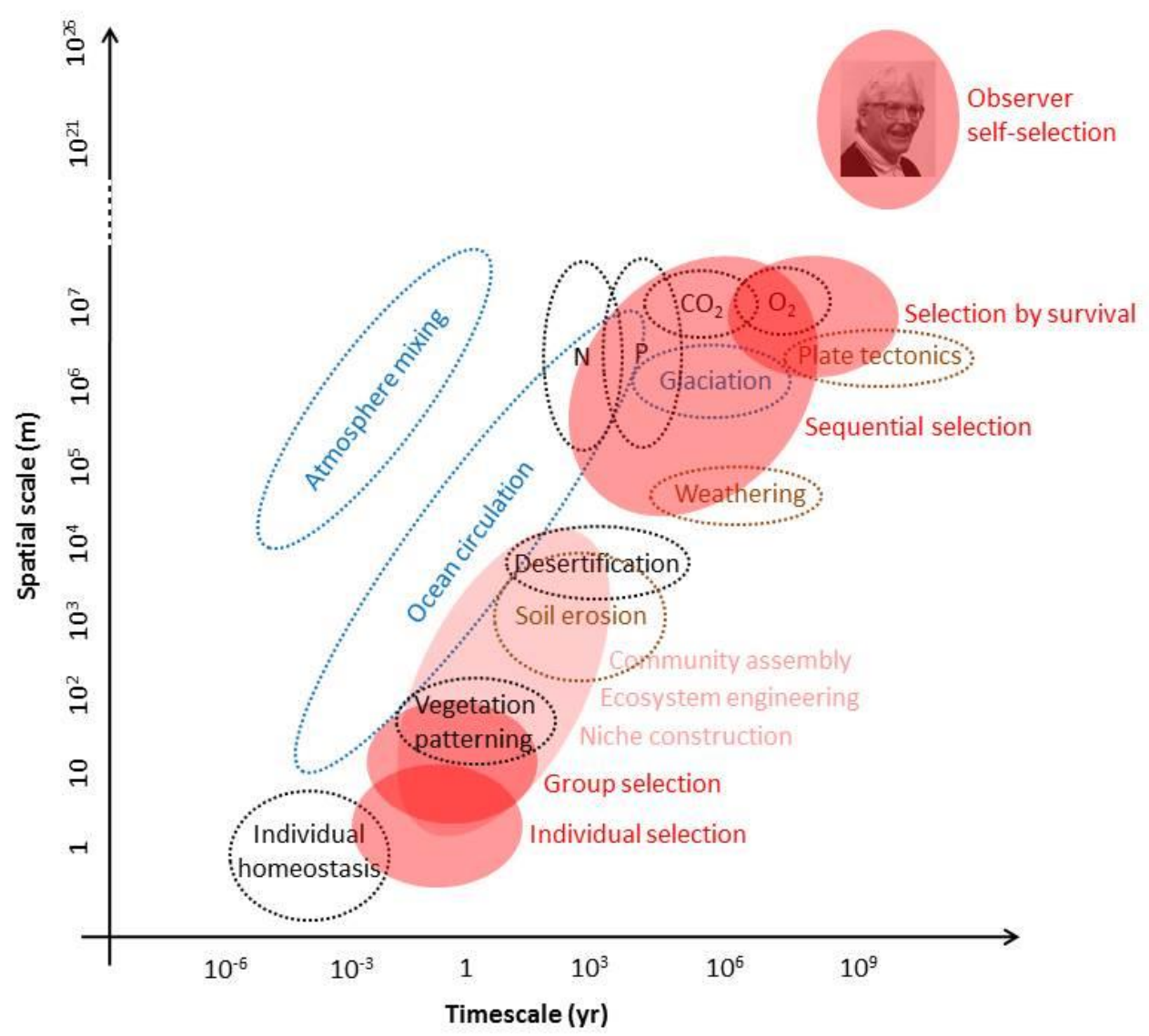

Figure 1. Space and time scales of Earth system processes and selection mechanisms.

Some key Earth system processes are shown in blue (ocean-atmosphere) and brown (land-

lithosphere). Examples of feedback mechanisms and regulated variables are shown in black.

Selection and self-organisation mechanisms are shown in red, with those described in the main text shown in darker red. The important evolutionary and/or ecological mechanisms of niche construction, ecological engineering and community assembly (pale red) can play a role in environmental regulation at intermediate space and time scales but are not reviewed

711 here due to space constraints and extensive coverage elsewhere. Note the break in the spatial scale bar from the scale of the planet $\left(\sim 10^{7} \mathrm{~m}\right)$ to galaxy $\left(\sim 10^{21} \mathrm{~m}\right)$ and universe

$713\left(\sim 10^{26} \mathrm{~m}\right)$ scales over which observer self-selection would operate. 


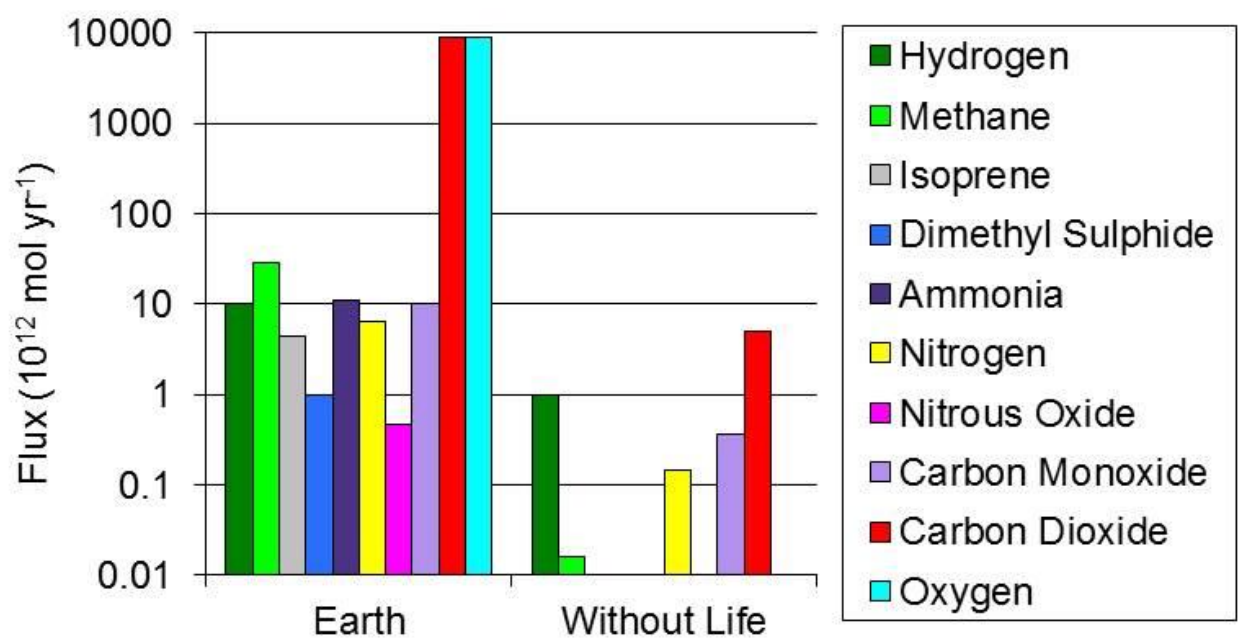

715 Figure 2. Gaseous recycling by Earth's contemporary biosphere. Fluxes of gases exchanged

716 at the surface of the Earth today and those estimated on an Earth without life

717 (corresponding to present day fluxes from volcanic and metamorphic processes) - as an

718 illustration of the remarkable recycling by today's biosphere - after Lenton [4]. 


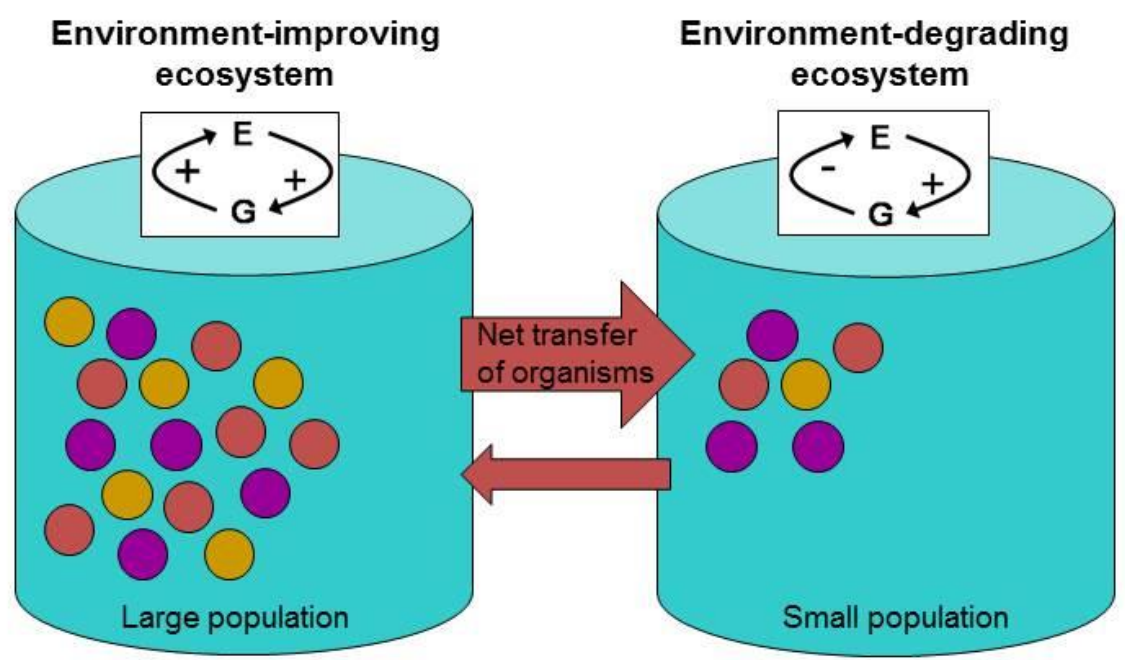

Figure 3. Ecosystem-level selection for the regulation of heterogeneous environmental variables. Illustration of the mechanism seen in the spatial Flask model of a network of microbial ecosystems (Box 1), where all the model 'microbes' share the same growth (G) response to the environment $(E)$, but have different nutrient requirements and different environmental effects [26]. Communities whose aggregated effect on their environment enhances their growth (left flask) have larger steady-state populations than communities whose collective effect degrades their environment (right flask). Denser populations in turn are better colonisers of available space and spread at the expense of less dense ones. The spread of environment-improving ecosystems alters the global environment toward (shared) optimal growth conditions. 


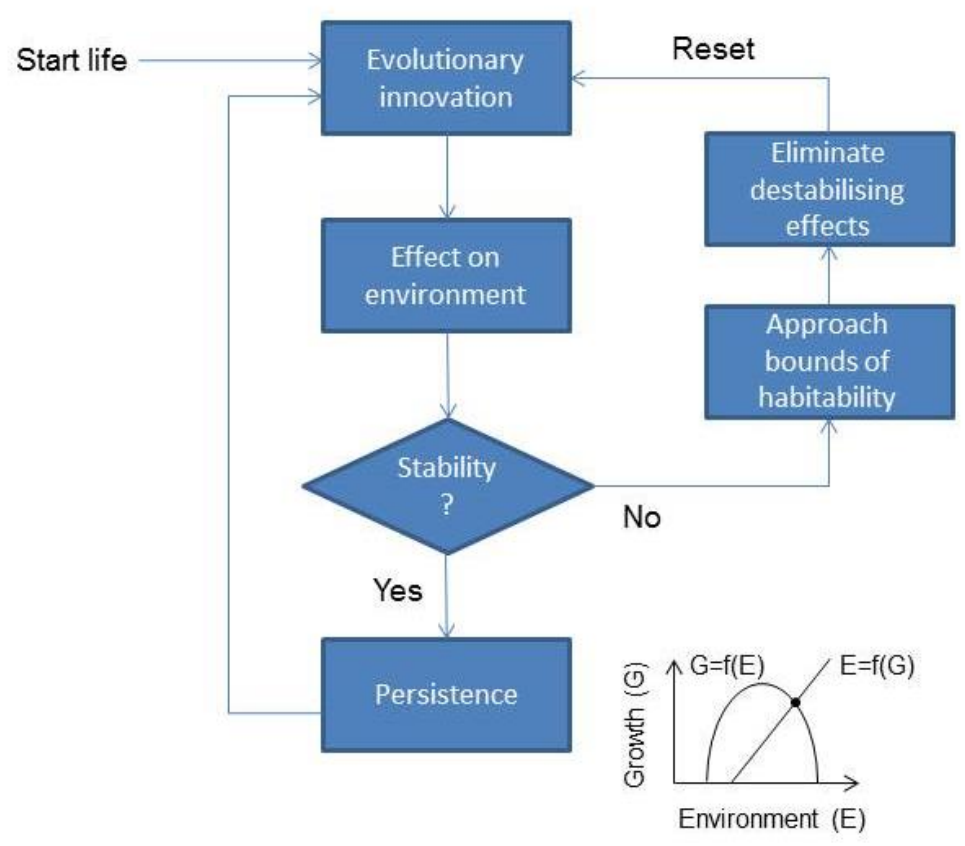

Figure 4. Sequential selection algorithm applied to the Earth. Assume that life starts somehow. Evolutionary innovation will then inevitably lead to environmental effects. Effects of life on the environment, interacting with abiotic feedbacks, could lead to a stable or an unstable outcome. If the environmental effects of life are destabilising, the system might then approach the bounds of habitability, e.g. in a 'snowball Earth' event. This effectively resets the system, eliminating the destabilising effects (and much else besides), and allowing a new (sequential) repetition to unfold. Again the effects of life on the environment might be stabilising or not, and if not the cycle can repeat itself. However, if the environmental effects of life are stabilising the resulting state will by definition tend to persist: A schematic example is given in the bottom right plot of growth (G) and environment (E) coupling - illustrating a single stable fixed point (black dot) in a negative feedback regime (i.e. a stable attractor). Such states will persist until destabilised by e.g. external forcing or further evolutionary innovation - which restarts the search algorithm for a stable configuration. 


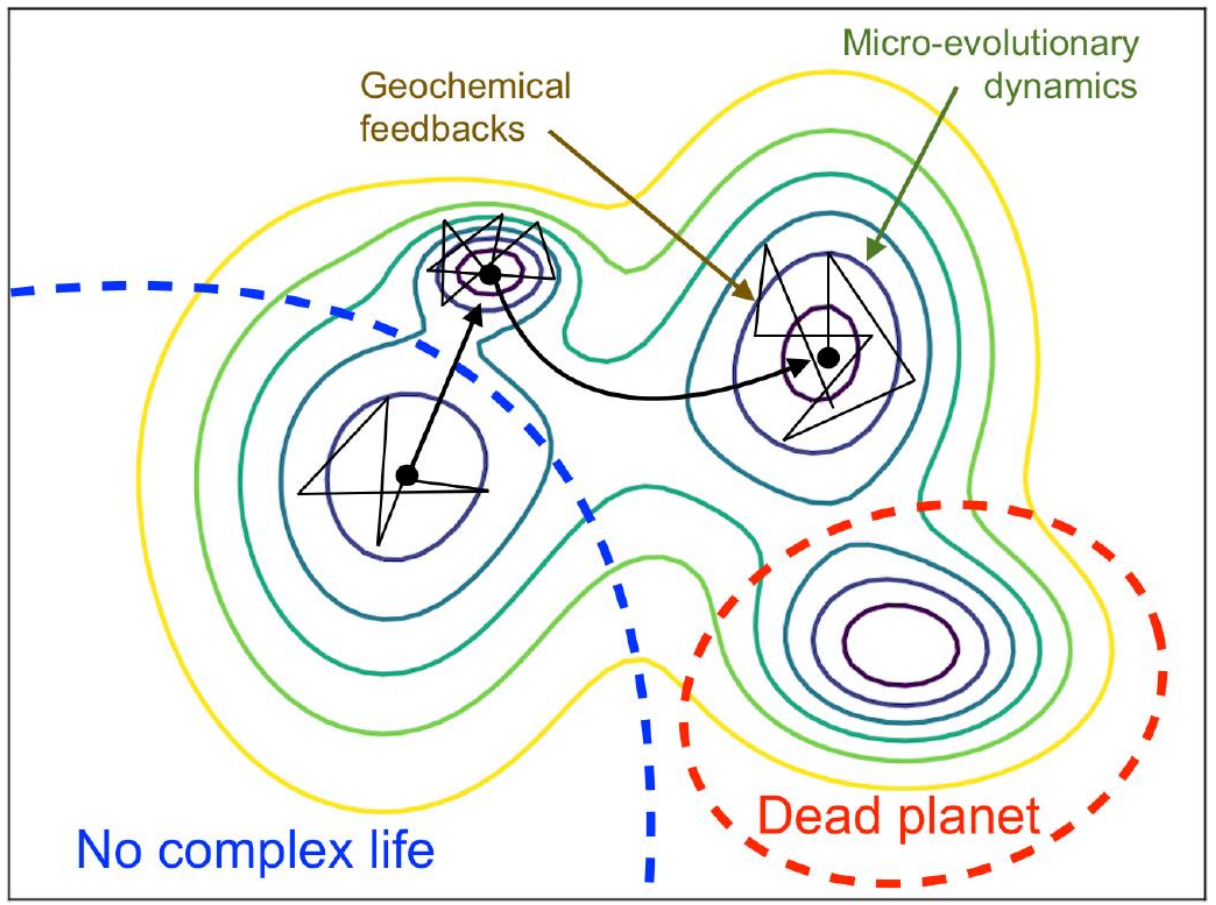

Figure 5. Visual metaphor for a sequential selection journey through the phase-space of a

complex system, here applied to a planet. The coloured contours map out a potential

surface that here includes four stable attractors (wells) denoted by shrinking concentric

rings that converge on a stable state (the bottom of each well). The trajectory of the system (over time) is indicated by the black lines and arrows. The system spends most of its time in stable states, with time proportional to the stability of the state. Destabilising evolutionary innovations drive rapid transitions (the arrows) through unstable regions. Stability is maximised where micro-evolutionary dynamics (green arrow) and underlying geochemical feedbacks (brown arrow) are aligned. External perturbations can also drive transitions to increasingly stable states. The system is presumed to start in an attractor that is not stable enough to support complex life but transitions to progressively more stable attractors. A highly stable but uninhabitable ('Dead planet') attractor also exists, which here might represent the aftermath of a 'runaway greenhouse' effect. Visual concept inspired by 